\title{
Regard et signification : la réception des peintures par le tracé d'annotation (Ikonikat)
}

Mathias Blanc

\section{OpenEdition}

\section{Journals}

Édition électronique

URL : http://journals.openedition.org/pa/976

DOI : $10.4000 /$ pa. 976

ISSN : 2273-0362

Éditeur

Université Lumière Lyon 2

\section{Édition imprimée}

Date de publication : 28 juillet 2020

Pagination : 58-74

ISSN : 1634-7706

\section{Référence électronique}




\title{
Regard et signification: la réception des peintures par le tracé d'annotation (Ikonikat)
}

\author{
Mathias Blanc \\ UMR 8529 IRHiS, CNRS/Université de Lille
}

\section{INTRODUCTION}

Dans le domaine expérimental des études visuelles au musée, la question «que voit-on dans une image? ? est traitée en se focalisant particulièrement sur les processus cognitifs via le suivi du regard (Rosenberg, 2013 ; Brinkmann et al., 2014). Les dispositifs oculométriques viennent ainsi alimenter un modèle de perception visuelle selon lequel le regard s'appuie sur des processus perceptifs de bas niveau (réagissant aux contrastes, aux couleurs, et aux formes) interagissant, notamment dans le cas de peintures, avec des processus de haut niveau (mobilisant l'entraînement et les connaissances artistiques préalables du regardeur) (Sparrow, 2019). Dans cette perspective, les résultats des études des mouvements oculaires suggèrent à la fois que, selon les styles de peintures, les modalités de découverte des œuvres sont favorisées par les savoirs pratiques déployés par l'observateur, mais aussi que la touche de l'artiste semble orienter les modalités perceptives du regardeur (Kapoula et al., 2015).

Néanmoins, autant la relation entre fonctions cognitives et structure de l'œuvre est interrogée par ces travaux de référence, autant la relation avec l'environnement social et la situation de visionnage est mise en retrait. En effet, une visite en musée correspond à une situation sociale spécifique dans un espace de médiations (Gottesdiener et Davallon, 2013) ; les œuvres sont accrochées dans un lieu dédié, dans un certain ordre, accompagnées d'un discours les présentant ; quant aux visiteurs, ils se distinguent de part leur âge, leur sexe, leurs savoirs pratiques et nous observons différentes configuration sociales de visite (en famille, en couple, entre amis, en groupe scolaire, en voyage organisé). Minimiser ces variables demeure problématique car cela aboutit à une vision abstraite de la visite muséale, déliée de son ancrage situationnel. Par contre, prendre en considération ces variables implique de développer des méthodes permettant de saisir leur signification pour l'activité de réception des œuvres par les publics. Finalement, comment ces facteurs socio-culturels peuvent-ils être identifiés et dans quelle mesure sont-ils significatifs pour le regard des visiteurs face aux œuvres?

Pour étudier ces questions, les démarches qualitatives en sciences sociales font davantage appel à la parole et à l'écriture, en demandant au regardeur de décrire ce qu'il voit, pour transformer le signal visuel en élément sémantique. 
Or le fait de faire appel au langage par le biais de la verbalisation pose à son tour un souci, notamment souligné par les recherches ayant pour objet l'image, la visualité et les pratiques visuelles. En effet, depuis les années 19901, nous observons un décentrage épistémologique pour interroger la spécificité $\mathrm{du}$ visuel; ces travaux se cristallisent autour d'une transformation paradigmatique, qualifiée d'Iconic turn ou de Pictorial turn suivant les aires intellectuelles (Boehm, 1994; Mitchell, 1994; Boehm et Mitchell, 2009) accompagnant un déplacement des modèles d'analyse sémiotiques et linguistiques. En d'autres termes, le recours à la verbalisation induirait une distorsion catégorielle de la réception des images.

Dans ce contexte, l'approche que nous développons avec la méthode du tracé d'annotation assistée par l'outil numérique Ikonikat (Ikonik Analysis Toolkit), consiste à explorer une voie supplémentaire pour compléter les démarches de suivi du regard par oculométrie ${ }^{2}$ (et éviter de subsumer les mouvements oculaires sous la signification) et celles d'attribution de sens par le texte ou la parole. Il s'agit de demander aux regardeurs d'interagir physiquement avec l'image par le geste, en montrant ce qu'ils perçoivent ou souhaitent décrire. Pourquoi demander à un public de dire ce qu'il voit, alors que nous pouvons lui demander de nous le montrer ? Cela revient à soutenir par l'action l'expérience visuelle des regardeurs, sans leur demander d'avoir recours immédiatement au langage verbal ou textuel, et cela facilite d'autant les comparaisons inter-individuelles et inter-culturelles en contournant les barrières linguistiques. Certes, l'influence $\mathrm{du}$ geste sur l'engagement $\mathrm{du}$ visiteur dans l'action doit être approfondi avec d'autres études futures, néanmoins, l'acte de monstration mobilise des savoirs partagés et partageables, et à ce titre, étudier ce que chacun perçoit et communique visuellement revient à analyser les normes véhiculées par l'image en contexte et leur degré de propagation.

\section{LA RECÉPTION DES PEINTURES EN CONTEXTE MUSÉAL}

\footnotetext{
${ }^{1}$ Notamment en Allemagne et dans les pays de langue allemande, cette effervescence s'inscrit dans le prolongement de problématiques qui structurent les thématiques visuelles depuis plus de vingt ans dans le monde académique germanophone. Les années 1990 ont ainsi vu apparaître un nouveau champ de recherche, la Bildwissenschaft, qui cherche à combiner les perspectives disciplinaires de l'histoire de l'art, des sciences de la communication et de la philosophie (Bredekamp, 2003 ; Belting, 2004). De manière concomitante, l'espace angloaméricain voit se développer des Visual Studies qui visent à décentrer les objets de recherche en histoire de l'art vers une prise en considération des cultures visuelles du quotidien (Mirzoeff, 1999 ; Evans et Hall, 1999).

${ }^{2}$ Notons que les dispositifs oculométriques nécessitent un temps certain pour être déployés et ne se prêtent pas actuellement à une utilisation de masse.
} 
A la suite des travaux précurseurs de Pierre Bourdieu (1965), les approches francophones traitent avec sagacité des usages sociaux des images ${ }^{3}$. Néanmoins, elles tendent à minimiser le questionnement sur l'image en tant qu'objet regardé. Cette dimension est effectivement davantage considérée par les études de réception, celles-ci visant à objectiver, à partir de l'expérience visuelle des regardeurs, " les actes sémiques de description, d'exploration, ou de segmentation d'une peinture qui sont directement en rapport avec l'iconographie ou la structure formelle d'une œuvre particulière » (Passeron, 2006 : 405).

Pour autant, notons que cela ne signifie pas qu'il nous faille circonscrire la réception d'une peinture à une approche sémiologique. Quand bien même la sémiologie développée par Roland Barthes concernant l'image fixe ${ }^{4}$ (1980) nous a sensibilisés aux diverses possibilités de signification de l'image, elle n'épuise pas le sens de l'œuvre regardée en situation; la réception des peintures apparaît indissociable du moment et de l'environnement dans lequel elles sont regardées.

En effet, certaines recherches empiriques permettent de saisir les liens entre action sociale située et production visuelle de sens. En situation expérimentale, de récents travaux en sciences cognitives soulignent l'importance du contexte et de l'intentionnalité du regardeur sur l'activation de traits sémantiques dans l'image (Kalénine, 2015). Ces recherches font écho aux études ethnométhodologiques qui mettent en lumière l'importance de la séquentialité des situations sociales, des savoirs pratiques incorporés, des jeux de différenciation sociale dans le déploiement du sens par l'image (Blanc, 2013), une prise en compte accrue de la structure séquentielle du déroulement empirique de l'action de regarder (Mondada, 2011), de la dimension communicationnelle de celle-ci (Knoblauch, Reichertz, Keller, 2012) et de sa combinaison historique avec des objectivations, c'est-à-dire des savoirs, des dispositifs et des institutions (Traue, 2012). A la suite de ces travaux, il paraît délicat d'étudier le sens attribué à une image regardée sans le lier aux configurations temporelles et sociales dans lesquelles il se déploie et qu'il contribue à définir.

Cet aspect est d'autant plus important que dans le domaine des études muséales, la question de l'accompagnement de visite nécessite d'être intégré à l'analyse des pratiques des visiteurs. En effet, les venues au musée sont effectuées en groupe, à une large majorité, ${ }^{5}$, et cet accompagnement a, notamment pour les publics les moins familiers avec cet environnement, une fonction de récréation, de réassurance (de mise en confiance) et d'enrichissement mutuel (Debenedetti, 2003). De surcroit, les recherches

\footnotetext{
${ }^{3}$ Par exemple, Gunthert, 2015.

${ }^{4} \mathrm{Ou}$ Christian Metz à propos du film cinématographique (Metz, 1968 et 1972).

${ }^{5}$ Dans l'étude à laquelle nous faisons référence par la suite, il est question de $96 \%$ de la population concernée; et en moyenne au niveau national, cela concerne plus de $85 \%$ des visites muséales (Eidelman, Jonchery et Zizi, 2012). Sur l'importance de cette dimension sociale de la visite, voir les travaux de Gottesdiener, 1990 ; Eidelman et Raguet-Candito, 2002 ; Debenedetti, 2003 ; Packer et Ballantyne, 2005 ; Eidelman et Roustan, 2008.
} 
exploitant des enregistrements vidéo des interactions sociales au musée mettent en avant l'importance de l'engagement des corps en co-présence sur la conduite de l'action (Vom Lehn, Heath, Hindmarsh, 2017). L'activité muséale apparaît profondément marquée par ces interactions en situation; dès lors, prendre en considération ce que nous pouvons appeler la configuration sociale de visite devient nécessaire pour étudier dans quelle mesure celle-ci joue un rôle dans la réception des œuvres.

Ce faisant, l'acte de regarder est à interroger comme pourvoyeur de sens : un sens suscité par une mise en résonnance de la configuration sociale (où et avec qui l'on regarde), de la trajectoire sociale et de l'imaginaire de celui qui regarde (ses représentations incorporées et projetées) avec l'immanence de la structure de l'image regardée (ce qu'elle m'autorise à voir ou à déceler). Mais par voie de conséquence, quelle méthodologie adopter pour tester cette approche ? Si l'on souhaite étudier les pratiques sociales qui se développent en situation réelle face aux images, c'est-à-dire sans les dissocier des situations dans lesquelles elles sont regardées, comment procéder?

\section{LA MISE EN GUVRE DU TRACÉ D'ANNOTATION}

De manière à disposer d'une méthode permettant de tenir davantage compte de cette spécificité visuelle et sociale, nous avons conçu l'Ikonik Analysis Toolkit (Ikonikat) permettant aux regardeurs d'annoter une image en traçant des courbes ou des segments sur une reproduction numérique de celleci. Les possibilités offertes par le tracé pour annoter ou expliquer une image ont particulièrement été mises en valeur par l'historien de l'art Max Imdahl (1996). Ses travaux, incontournables Outre-Rhin, ont également été disséminés dans les disciplines connexes, les sciences sociales germaniques (Bohnsack, 2009; Breckner, 2012). Néanmoins, ces différentes approches limitent l'exploitation du tracé à la communication entre spécialistes de l'image. Autrement dit, l'utilisation du tracé d'annotation pour permettre à un public non-spécialiste de communiquer sur sa réception des images n'a pas encore été envisagée. Or, si l'on propose à un public de tout âge, ne disposant pas du vocabulaire technique adéquat pour analyser une image, de désigner ce qu'il voit, de nouvelles perspectives s'ouvrent pour étudier la réception des visuels, tant sur le plan individuel (perceptif et cognitif), que sur le plan social et culturel (Blanc, 2017).

Donner la possibilité à chacun de montrer ce qu'il pense pertinent de voir dans l'image, sans disposer d'un vocabulaire spécifique, a imposé une approche pluridisciplinaire (sciences cognitives et sciences sociales) et le développement d'outils numériques exploitant les capacités communicationnelles du dessin. Ceci a impliqué d'envisager une récolte participative des données et ensuite de mettre au point des modalités de traitements qui tiennent compte de la richesse des informations transmises. 
Concrètement, au musée, arrivant face aux œuvres, les visiteurs sont invités à se munir de tablettes tactiles sur lesquelles s'affiche une reproduction numérique de la peinture qui leur fait face. Il leur est ensuite demandé de désigner par le tracé (lignes, courbes, points) ce qui attire en premier leur regard, puis ce qu'ils estiment saisissant dans l'œuvre. Le regardeur est ainsi amené à pointer, relever ou détourer des éléments perçus dans la peinture, en dessinant des segments ou des courbes sur des tablettes. Nous proposons ainsi au visiteur d'ajouter, sur l'image observée, des tracés désignant les éléments picturaux qui lui semblent essentiels ou qui l'interrogent (Photographies 1 et 2).

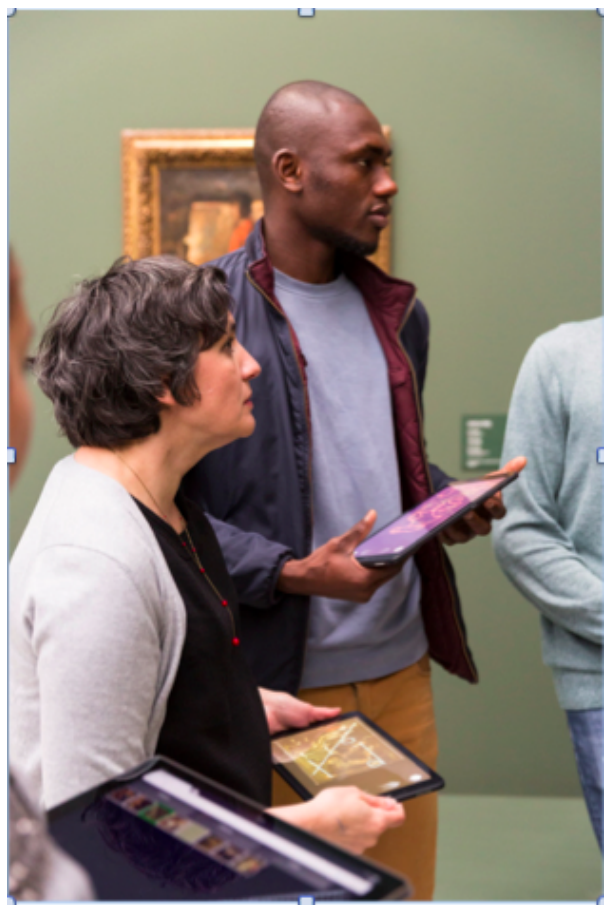

Photographie 1 : Visiteurs exploitant Ikonikat au musée du Louvre-Lens, juin 2017 (C) Claire-Lise Havet/Musée du Louvre-Lens/IKONIKAT/CNRS Photothèque

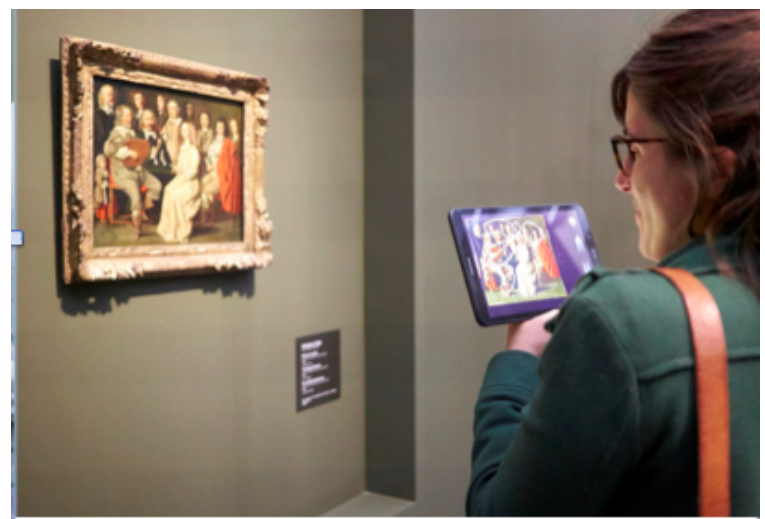

Photographie 2 : Annotation avec Ikonikat face à la Réunion musicale d'Antoine Le Nain (C) Claire-Lise Havet/Musée du Louvre-Lens/IKONIKAT/CNRS Photothèque 
Le procédé est exploité face aux différentes œuvres du parcours muséal en respectant le rythme de visite de chacun. Les actions sur les tablettes sont visualisables en temps réel et les utilisateurs peuvent présenter leurs tracés d'annotation à l'ensemble du groupe pour entamer, s'ils le veulent, une discussion collective face aux œuvres. A la fin de leur parcours, les participants remplissent un questionnaire textuel en fournissant des informations sociographiques (âge, sexe, formation, habitude de fréquentation des musées etc.) $)^{6}$.

En passant par le tracé, Ikonikat offre la possibilité de retarder le moment de l'énonciation verbale face aux œuvres et, par conséquent, de soutenir l'expérience esthétique visuelle du visiteur ${ }^{7}$; lorsque les publics s'expriment d'eux-mêmes sur les annotations graphiques qu'ils viennent de réaliser, ces verbalisations sont consignées dans un carnet de terrain et servent par la suite à catégoriser les tracés recueillis. Cette typification s'appuie également sur une visualisation de la densité des tracés sur les œuvres grâce à la réalisation de cartes de chaleur (heatmap) des tracés. En parallèle, de manière à traiter l'ensemble des données recueillies, une classification des tracés est effectuée à l'appui d'un algorithme d'apprentissage. Le traitement des tracés articule donc une approche qualitative de typification et une méthodologie quantitative de classification.

\section{L'ÉTUDE DE RÉCEPTION DE L'EXPOSITION «LE MYSTÈRE LE NAIN » AU MUSÉE DU LOUVRE-LENS}

Au printemps 2017, de mars à juin, la méthode Ikonikat a été déployée à grande échelle au musée du Louvre-Lens. Face aux peintures des frères Le Nain, 750 visiteurs ont ainsi fait part de leur expérience de visite. L'échantillon de notre étude a été constitué de manière aléatoire, de manière à minimiser les biais de la méthode des quotas et à nous assurer de sa plus forte représentativité possible. Ainsi, notre effectif compte 36\% de scolaires, $31 \%$ de personnes venues en famille, $22 \%$ de personnes en couple ou entre amis, $7 \%$ de personnes en voyage organisé et $4 \%$ de personnes seules; les femmes représentent deux tiers des effectifs pour les plus de 25 ans; et en termes d'âge, $41 \%$ de l'effectif global a moins de 18 ans, 8\% entre 18 et 25 ans, 30\% entre 25 et 60 ans, et $31 \%$ de l'échantillon a plus de 60 ans. La prise en compte de cette sociographie est nécessaire à l'analyse des résultats qui va suivre.

\footnotetext{
${ }^{6}$ Les indicateurs sont communs à ceux exploités lors d'études antérieures sur les publics (Eidelman et Jonchery, 2011).

${ }^{7}$ Concernant l'influence des travaux de l'historien d'art Max Imdahl sur cette perspective, voir Blanc (2017). A propos d'Ikonikat (http://ikonikat.org), voir l'article consacré dans CNRS Le journal de mars 2017, et la vidéo disponible en ligne : https:/ / lejournal.cnrs.fr/videos/comment-regarde-t-une-oeuvre-dart
} 
L'intitulé de l'exposition "le Mystère Le Nain »se référait à la question épineuse de l'attribution de ces œuvres du XVII ${ }^{e}$ siècle : lequel des trois frères Le Nain, travaillant dans le même atelier, a peint telle peinture ou a davantage influencé, par son geste, l'expression picturale de telle œuvre ? Leurs tableaux ne portent pas ou peu la marque de leur provenance ; lorsqu'une signature apparaît, elle est réduite au seul patronyme des peintres. Par conséquent, les commissaires de l'exposition, Nicolas Milovanovic et Luc Piralla, proposaient un parcours scindé en plusieurs étapes, commençant par deux salles sensibilisant à cette problématique puis s'organisant en plusieurs espaces, le premier étant consacré au geste de Louis, le second dédié à Antoine et le troisième dévolu à Mathieu. Plusieurs peintures inspirées de celles des Le Nain étaient ensuite présentées aux visiteurs et l'exposition se terminait par des œuvres dont l'attribution est toujours controversée (Milovanovic et Piralla-Heng Vong, 2017). De fait, la muséographie invitait les visiteurs à percer le mystère de l'attribution mais, pour autant, dans quelle mesure les publics l'ont-ils perçu?

En concertation avec les commissaires de l'exposition, un corpus de sept œuvres emblématiques de la perspective du commissariat fut constitué. Les trois premières œuvres étaient attribuées à Louis Le Nain et deux d'entre elles correspondaient aux scènes de genre paysannes qui ont fait leur réputation ${ }^{8}$; la quatrième était une miniature sur cuivre d'Antoine Le Nain ${ }^{9}$; les cinquième et sixième concernaient les peintures religieuses de Mathieu Le Nain ${ }^{10}$; et la dernière figurait un portrait de groupe qui divise les experts quant à son attribution aux frères Le Nain ${ }^{11}$. Au musée, après avoir pénétré dans les premières salles et arrivant face à la première œuvre du corpus, les visiteurs étaient invités à se munir de tablettes tactiles sur lesquelles s'affichait une reproduction numérique de la peinture qui leur faisait face et à annoter par le tracé les aspects qui retenaient leur attention dans la peinture regardée. Au final, les 33500 tracés récoltés au cours des 60 jours d'enquête ont ainsi pu faire l'objet d'une analyse intégrant les informations sociographiques transmises par les 750 visiteurs âgés de 4 à 93 ans ayant participé à l'étude.

Nous pouvons remarquer que les tracés d'annotation recueillis se répartissent en deux groupes : le premier concerne $77 \%$ des tracés et met en valeur une lecture iconographique des œuvres, alors que le second, avec $23 \%$ des tracés, témoigne d'une approche plastique des peintures (Figure 1).

\footnotetext{
${ }^{8}$ Pour les scènes de genre attribuées à Louis Le Nain, il est question de Famille de paysans dans un intérieur (1642), huile sur toile, $113 \times 159 \mathrm{~cm}$, RF 2081 ( $\mathrm{n}^{\circ}$ photo 08-551310) et Repas de paysans (1642), huile sur toile, 97x122 cm, MI 1088 (n ${ }^{\circ}$ photo 15-615803). La troisième correspond à $L a$ Forge (1642-1643), huile sur toile, $69 \times 57 \mathrm{~cm}$, INV 6838 (nº photo 15-615516).

9 Antoine le Nain, la Réunion musicale (1642), huile sur cuivre, 32x40 cm, RF 1067(nº photo 11525157).

${ }^{10}$ Mathieu Le Nain, Les pèlerins d'Emmaüs (1645), huile sur toile, 75x92 cm, RF 1950-8 ( ${ }^{\circ}$ photo 12-548172), et Le reniement de Saint Pierre (1648), huile sur toile, 95x122 cm, RF 2008-56 $\left(\mathrm{n}^{\circ}\right.$ photo 10-501241).

${ }^{11}$ Indéterminé, L'Académie (1640), huile sur toile, 116x146 cm, RF 701 (nº photo 16-513906).
} 

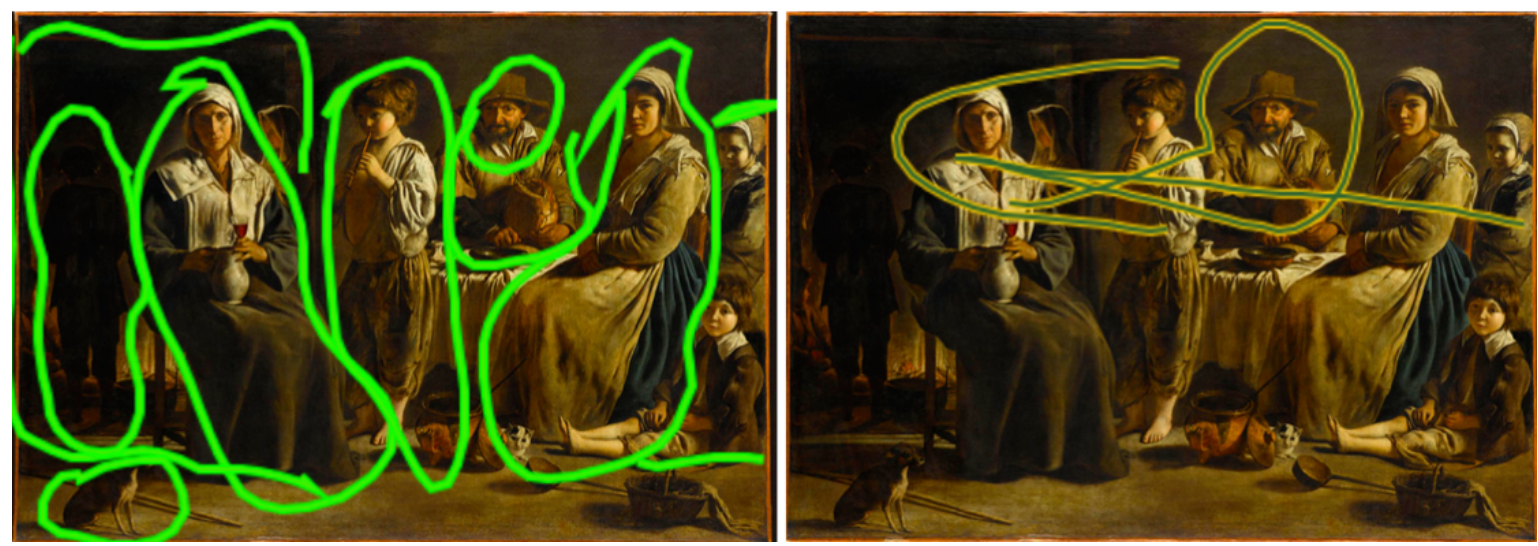

Figure 1 : Tracés d'annotation iconographique (gauche) et d'annotation plastique (droite) (C) RMN-Grand Palais (musée du Louvre)/Angèle Dequier/Ikonikat/CNRS

La dimension iconographique des annotations est révélée par des tracés détourant des personnages, des animaux, des objets ou des détails de ceux-ci. $\mathrm{Au}$ contraire, les lignes droites ou les lignes courbes, qui ne se referment pas sur elles-mêmes et ne détourent pas des personnages ou des objets, témoignent d'une mise en relation plastique des éléments picturaux. Cette différence entre les tracés réalisés nous fournit ainsi rapidement une information sur le type de savoir mobilisé par les visiteurs. La reconnaissance de motifs concerne ainsi les trois quarts des tracés mais se révèle surtout être majoritaire à $86 \%$ pour les visiteurs profanes (ne disposant pas de formation artistique ou n'ayant pas suivi de cursus en histoire de l'art). A contrario, les visiteurs experts utilisent autant les annotations plastiques qu'iconographiques. Cette variation est corroborée par le contenu des verbatims des visiteurs: les visiteurs profanes évoquent moins les aspects plastiques des œuvres que les experts. A ce titre, la distinction entre les types de tracés d'annotation procède pleinement du caractère social de l'espace muséal, son expression peut aussi être perçue comme un marqueur de distinction sociale entre profanes et experts. Cet aspect mérite d'être approfondi par d'autres recherches mais l'hypothèse invite à ne pas oublier que notre méthode se concentre avant tout sur la dimension sociale du regard en situation.

En effet, rappelons que la méthode du tracé d'annotation assistée par Ikonikat a été conçue dès le départ comme une méthode complémentaire à celles existantes, et ceci nécessite de les comparer. Les analyses face aux scènes de genre paysannes amènent des éléments permettant $d^{\prime}$ abonder dans ce sens. En l'espèce, autant l'importance des regards frontaux des personnages est soulignée grâce aux cartes de chaleur ${ }^{12}$ effectuées à l'aide d'Ikonikat (Figure 2), autant l'étude oculométrique réalisée en parallèle sur les mêmes œuvres fait

\footnotetext{
${ }^{12}$ Les cartes de chaleur servent à visualiser la fréquence de répartition des tracés d'annotation sur l'œuvre. Plus la zone est rouge, plus elle est partagée par les utilisateurs d'Ikonikat.
} 
apparaitre ces regards frontaux comme étant de même intensité que d'autres regards et éléments scéniques (Figure 3$)^{13}$.
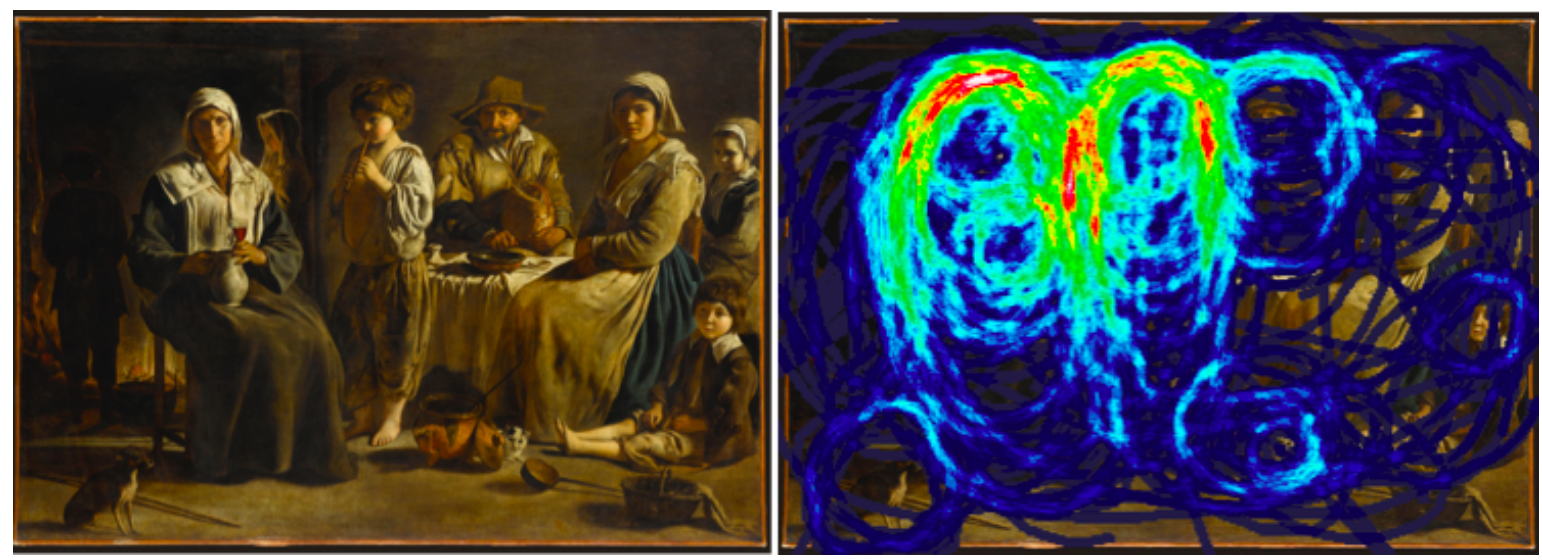

Figure 2: Carte de chaleur des tracés effectués par les visiteurs sur Famille de paysan de Louis Le Nain

C RMN-Grand Palais (musée du Louvre)/Angèle Dequier/Ikonikat/CNRS
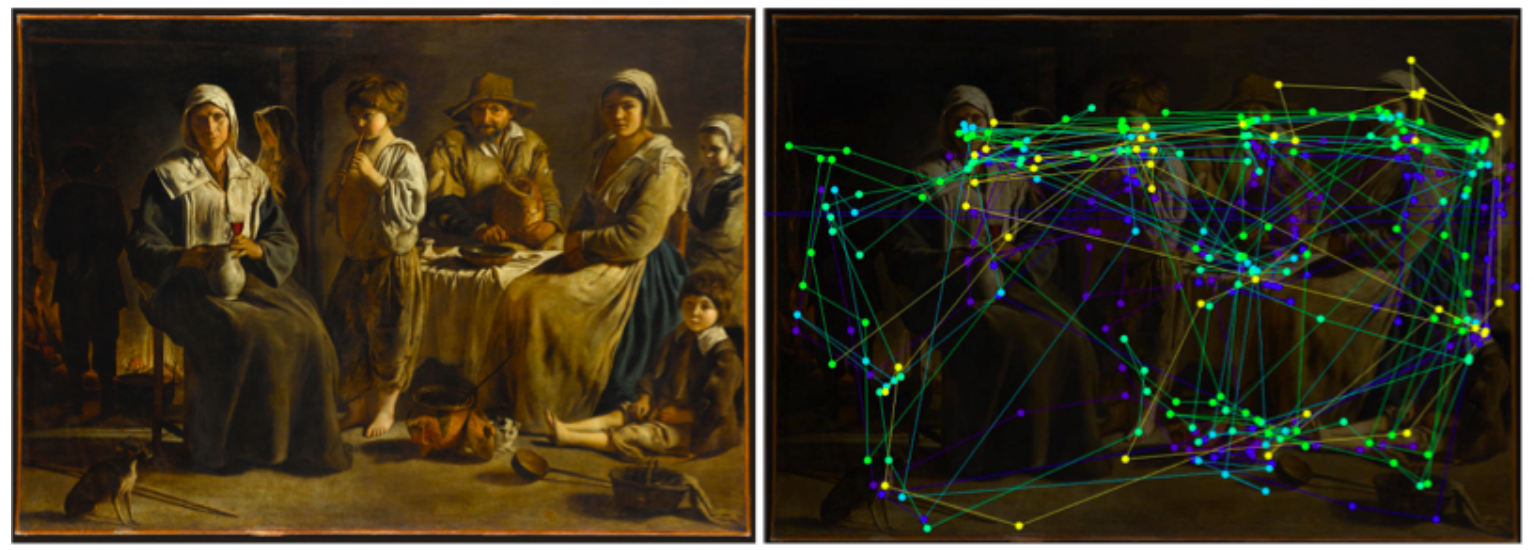

Figure 3 : Fixations et saccades oculaires relevées par oculométrie

(C) RMN-Grand Palais (musée du Louvre)/Angèle Dequier/Laurent Sparrow/Equipex IrDIVE

Ces résultats peuvent sembler discordants mais si nous les regardons sous l'angle de la complémentarité, le croisement des deux approches mène à l'hypothèse d'un évitement oculaire de la part des visiteurs: en effet, les regards orientés vers le spectateur dans les scènes paysannes ne semblent pas spécifiquement soutenus par les visiteurs alors qu'ils leur attribuent une signification primordiale. D'ailleurs, après avoir tracé avec Ikonikat, qu'ils soient hommes, femmes, adultes ou enfants, les visiteurs verbalisaient un

\footnotetext{
${ }^{13}$ Plusieurs approches d'études du visuel ont été exploitées sur ce corpus d'œuvres et ont fait l'objet d'un colloque-atelier organisé en mars 2017 : http://visuall.hypotheses.org/colloqueatelier-international-regards-interdisciplinaires-sur-lexposition-le-mystere-le-nain-musee-dulouvre-lensinternationaler-workshop-interdisziplinare-betrachtungen
} 
sentiment de gravité face à cette œuvre et évoquaient une atmosphère pesante à son endroit. Cette mise en relation des méthodes interroge anthropologiquement autant notre culture contemporaine du regard que celle des frères Le Nain et de leurs acheteurs au XVII siècle ; comment regardaient ces peintres et leurs commanditaires ou acheteurs ? Nous ne disposons après tout que de peu d'indices pour répondre à cette question, et la réception contemporaine de ces œuvres invite à l'entretenir.

Un autre résultat propice à l'entretien des problématiques du champ muséologique concerne la question de l'influence de la muséographie sur la réception des visiteurs. A ce titre, la notion de «mystère » et la présentation de cette problématique en début de parcours semblent clairement avoir un effet sur le déploiement des regards en situation. Au mystère de l'attribution se rajoute un mystère du sujet, ce dont témoigne une attention progressive vers les motifs picturaux à l'arrière-plan des œuvres (Figure 4).
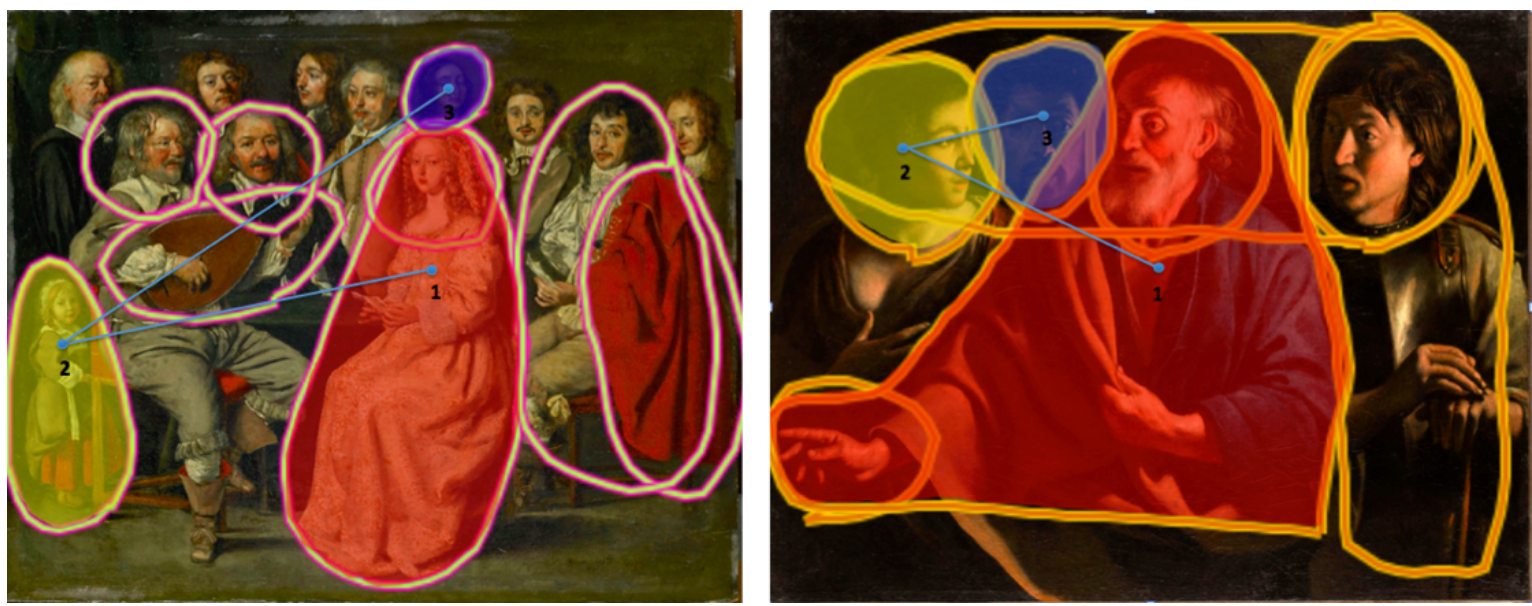

Figure 4 : Séquentialité majoritaire des motifs annotés par les visiteurs ayant suivi le parcours de visite dans le sens proposé par le commissariat de l'exposition

C RMN-Grand Palais (musée du Louvre)/Angèle Dequier/Ikonikat/CNRS

Nous voyons ainsi arriver, en troisième position des motifs les plus mis en avant par les visiteurs, des têtes ou des corps à l'arrière-plan. Ce phénomène se renforce en avançant dans l'exposition. Néanmoins, cette focalisation n'est pas observée si les publics effectuent la visite en sens inverse, c'est-à-dire s'ils ne bénéficient pas des informations contextuelles transmises dans les premières salles du parcours conçu par les commissaires de l'exposition. En d'autres termes, une formation du regard qui est liée à la muséographie s'effectue au cours de la visite. Ces résultats corroborent ceux d'Atsuko Kawashima et Hana Gottesdiener (1998) qui notaient que la séquentialité de l'accrochage avait un impact sur la reconstitution du fil conducteur de l'exposition par les visiteurs.

Toutefois, nous ne pouvons pas affirmer que le parcours d'exposition et le cadre proposé orientent de manière unidirectionnelle les regards des 
différents publics de visiteurs. Ainsi, une incompréhension des visiteurs concernant le titre de l'œuvre Réunion musicale amène d'autres interprétations, et la visualisation par Ikonikat du décalage entre la perception des publics profanes et celle des experts ouvre de nouvelles perspectives. Gardons à l'esprit que l'intitulé de cette peinture correspond à une interprétation experte postérieure à la réalisation des peintres et se réfère à l'avènement des airs de cour comme pratique de divertissement prisée des salons du xviie siècle. Or la séquentialité de motifs relevée par plusieurs publics présente cette œuvre comme une scène de fiançailles (Figure 5).
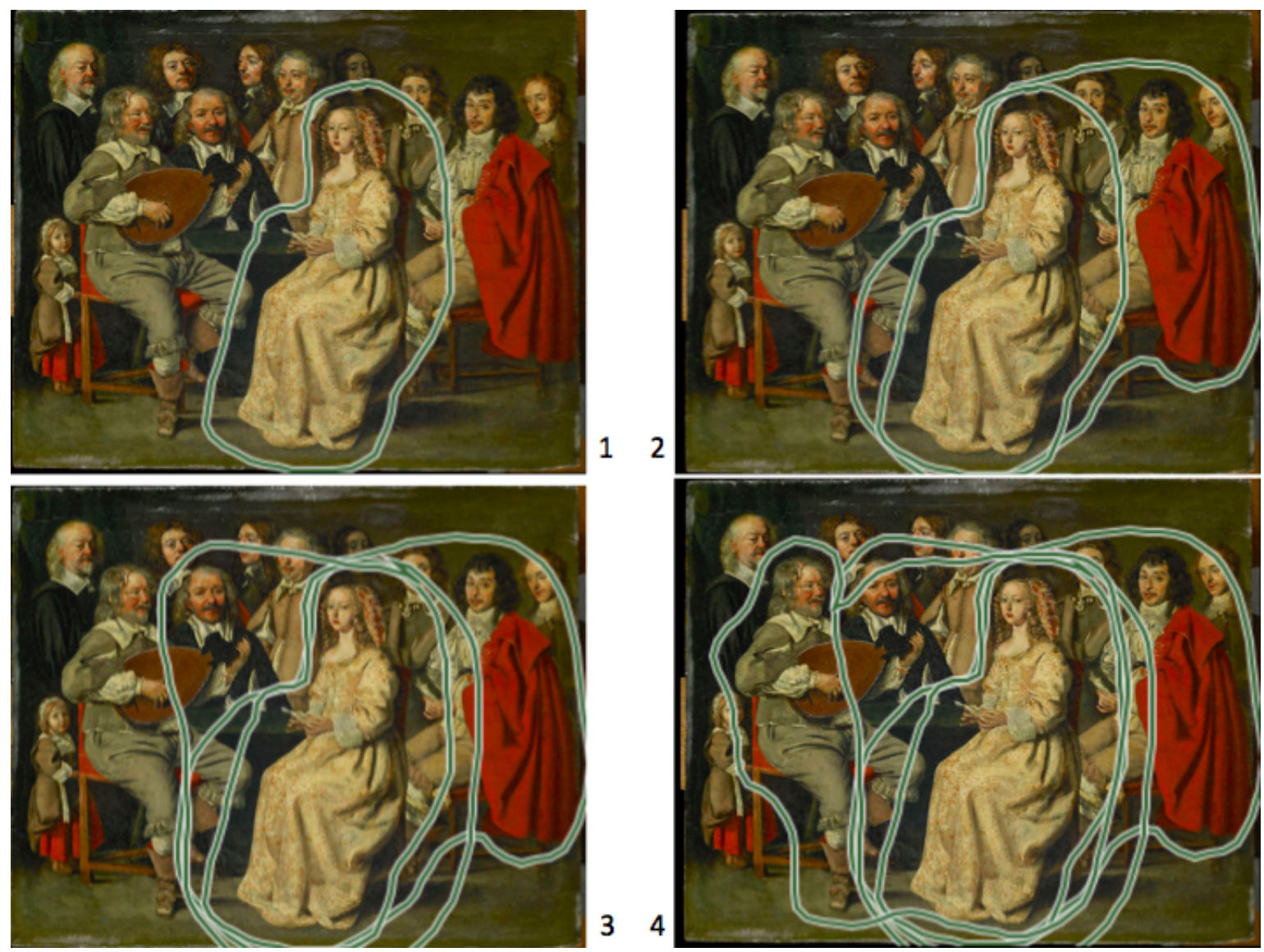

Figure 5 : Tracés-types révélant une interprétation de la scène comme une réunion de fiançailles (C) RMN-Grand Palais (musée du Louvre)/Angèle Dequier/Ikonikat/CNRS

Le premier détourage met en avant la jeune femme au centre de la peinture et la qualifie de "fiancée ", comme en attestent les verbatims ; le second crée un ensemble incluant la fiancée et l'homme à la cape rouge, son fiancé ; le troisième joint à la fiancée un homme aux cheveux grisonnants, vêtu de noir avec un col en dentelles, et serait le père de la jeune femme; quant au quatrième tracé, il intègre le musicien mais lui confère un rôle secondaire pour exprimer la dimension festive de la scène. 
L'organisation des tracés révèle ainsi une autre interprétation cohérente (organisée en séquences) et plausible (aucun document positif ne fournit d'intitulé à cette peinture) qui entre en dialogue avec le savoir expert. Nous ne soutenons absolument pas que des interprétations $\mathrm{du} \mathrm{XXI}^{\mathrm{e}}$ siècle puissent correspondre trait pour trait à des interprétations du XVII ${ }^{e}$ siècle mais, avec ce cas d'étude, nous constatons que des interprétations profanes peuvent proposer un décentrage propice à l'enrichissement des questionnements alimentant l'expertise. Cette dernière peut aussi se servir des interrogations profanes pour soutenir son attitude réflexive face à un socle de savoirs institutionnalisés.

Pour finir, notons que les traitements statistiques effectués soulignent la forte spécificité d'une variable dans l'organisation des motifs iconographiques, en l'occurrence celle du sexe des visiteurs. En effet, la classification des tracés permet d'étudier des séquences d'annotations à partir de l'ordre des tracés de chaque visiteur (par exemple pour la peinture Famille de paysans, il est question pour certains visiteurs des motifs "tête de la dame âgée à gauche », puis "verre de vin tenu par ce personnage », et ensuite "pain sur la table face à l'homme assis »). Les analyses factorielles entre ces séquences et les différentes variables sociographiques fournissent un résultat suscitant l'interrogation car les écarts à l'indépendance des modalités d'une variable en particulier sont marqués : ce sont les modalités «homme» et "femme» de la variable «sexe ». C'est-à-dire que dans l'espace des correspondances, une forte corrélation entre cette variable et l'organisation des tracés d'annotation est marquée.

En termes de regards portés sur ces peintures, diverses modalités perceptives peuvent ainsi être identifiées. Par exemple concernant le tableau Famille de paysans (Figure 6), nous observons davantage auprès des publics masculins une nette tendance à faire état d'une exploration des plans en spirale aboutissant à une focalisation sur des détails à l'arrière-plan, alors que le regard d'une majorité de femmes (visitant entre femmes) se concentre sur l'expression des visages et les postures des personnages centraux. 

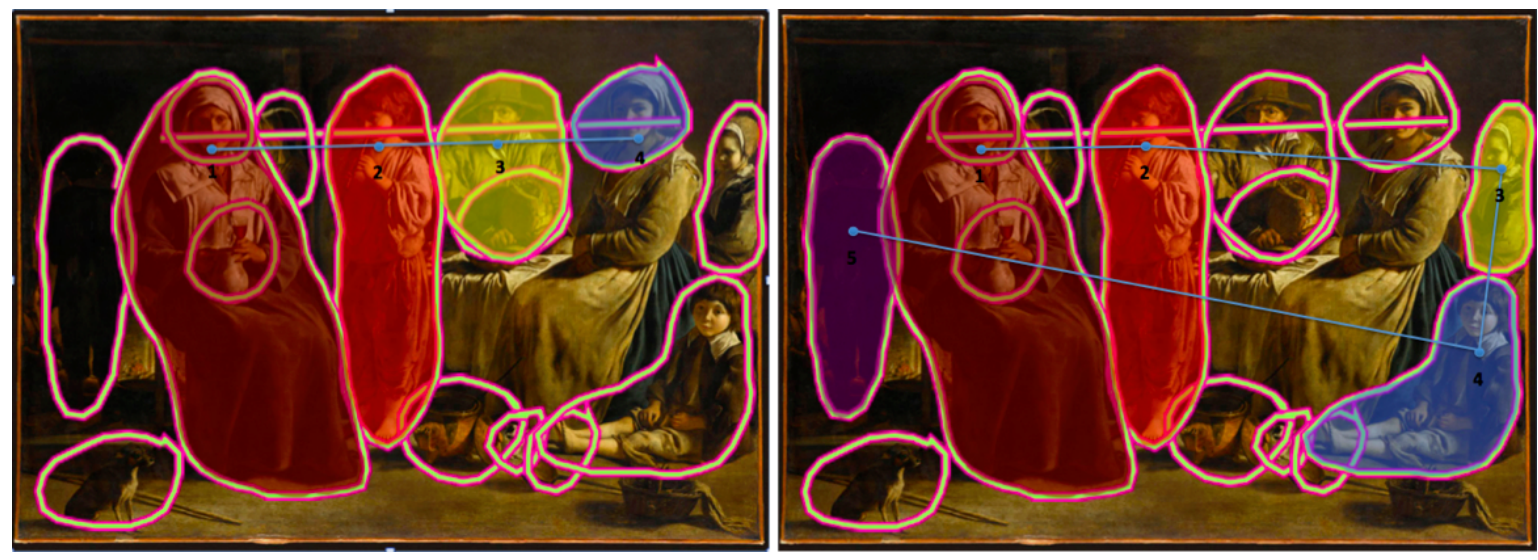

Figure 6 : Séquentialité majoritaire des tracés pour des groupes constitués uniquement de femmes (à gauche) et des groupes avec des hommes (à droite)

(C) RMN-Grand Palais (musée du Louvre)/Angèle Dequier/Ikonikat/CNRS

Cependant, ce résultat ne peut être interprété comme une stricte dichotomie homme-femme prévalant dans la réception des œuvres. En effet, les données étant forcément produites en contexte, en l'occurrence réalisées en situation de visite muséale, l'analyse de celles-ci impose de tenir compte des conditions dans lesquelles elles ont été récoltées et des caractéristiques de la population étudiée. De fait, l'importance de cette variable dans la réception des œuvres apparaît soutenue par plusieurs facteurs, à savoir l'âge, la configuration sociale de la visite (en famille, en couple, dans un cadre scolaire...) et la formation des visiteurs. Ainsi, rappelons que dans notre population, les femmes sont surreprésentées, elles sont deux fois plus nombreuses que les hommes à accéder à l'exposition (respectivement $65 \%$ et $35 \%$ ), phénomène particulièrement saillant chez les séniors où les visites exclusivement entre femmes sont majoritaires (62\%). Concernant les jeunes publics, près des deux tiers de l'effectif (68\%) correspondent à des enfants ayant découvert l'exposition dans un cadre scolaire, c'est-à-dire lors de visites au cours desquelles des sous-groupes de filles et d'autres de garçons se constituent distinctement. Quant aux tracés réalisés par les visiteurs compris entre 25 et 60 ans, la corrélation semble moins saillante pour ces publics. Ce qui peut s'expliquer par le fait que ceux-ci sont, pour moitié (53\%), réalisés dans un cadre familial davantage orienté vers la prise en compte de la perception de l'enfant ou des enfants accompagnant que par un accès immédiat aux œuvres.

En d'autres termes, nous ne pouvons statuer de manière univoque sur une distinction homme-femme qui s'observerait; il nous faut plutôt envisager que cette différence soit le résultat d'horizons de sens qui évoluent en fonction des personnes accompagnant la visite et avec lesquelles le visiteur développe son identité; en l'occurrence, il s'agit davantage de femmes entre elles qui se projettent dans une relation sociale avec des attentes identitaires. Ainsi, si l'on émet l'hypothèse d'un regard genré, ce serait en interrogeant cette dimension de phénoménologie sociale. Cette voie sera à suivre, et quand bien même nous 
travaillons à partir d'éléments recueillis auprès de regardeurs contemporains, elle peut servir à alimenter les débats sur le genre en histoire de l'art car la question de l'accompagnement porte également sur les configurations sociales $\mathrm{qu}^{\prime}$ envisageaient les artistes pour leurs œuvres ${ }^{14}$.

\section{CONCLUSION}

En somme, la méthode du tracé d'annotation assistée par Ikonikat interroge la manière dont les visiteurs de musée regardent les peintures exposées, cherche à cerner ce qu'ils voient, à identifier les ressources qu'ils mobilisent à cet effet, et vise à comprendre les modalités d'interaction qui se déploient en situation de visite.

En fonction de cette situation, de son organisation temporelle institutionnalisée et des enjeux de positionnement social des acteurs, différents traitements sériels de l'information picturale peuvent être relevés avec des images identiques. De fait, les arpentages et parcours visuels effectués lorsque l'on regarde une image, c'est-à-dire les chemins d'accès aux territoires délimités par celle-ci et les connaissances mobilisées, sont soumis à des variations ayant une signification fondamentalement sociale. D'où la nécessité de faire appel directement aux regardeurs et de recueillir leurs observations pour cerner ces dimensions multiples. A ce titre, une démarche participative de récoltes de données s'impose, tout en sollicitant la monstration et en retardant l'énonciation face aux peintures.

Les résultats d'une étude empirique de trois mois au musée du LouvreLens interrogent l'influence de la muséographie sur l'évolution du regard des visiteurs au fur et à mesure de leur parcours dans l'exposition. Ils permettent également d'identifier diverses interprétations des publics qui questionnent la présentation des œuvres. Ils mettent enfin en lumière différentes modalités de réception des œuvres qui varient notamment selon la formation pratique des visiteurs et la configuration sociale des visites, ce dernier facteur semblant soutenir une perception genrée des peintures des frères Le Nain. Ces éléments constituent autant de pistes de recherche à suivre pour cerner l'étendue des possibilités de la méthode du tracé d'annotation assistée par Ikonikat.

\footnotetext{
${ }^{14}$ Nicolas Milovanovic évoquait cette piste de recherche pour questionner la destination initiale des scènes de genre paysannes, en référence aux travaux de Diefendorf (2004) sur les femmes et la piété au début du XVIIe siècle.
} 


\section{BIBLIOGRAPHIE}

Roland BARTHES, La chambre claire, Paris, Gallimard, 1980.

Hans BELTING, Pour une anthropologie des images, trad. J. Torrent, Paris, Gallimard, 2004.

Mathias BLANC, "L'Iconique de Max Imdahl et sa fécondité pour le décloisonnement des savoirs/Max Imdahls Ikonik und ihr positiver Einfluss auf die Entgrenzung des Wissens », Regards croisés, Revue franco-allemande d'histoire de l'art et esthétique, No. 7, 2017, pp. 55-80.

Mathias BLANC, «Images de soi, images de foi. Sociologie visuelle d'un rassemblement pontifical », Revue des Sciences sociales, n49, 2013, pp. 20-25

Gottfried BOEHM, « Die Wiederkehr der Bilder », in Gottfried BOEHM (dir.), Was ist ein Bild?, Munich, Wilhelm Fink Verlag, 1994, pp. 11-38.

Gottfried BOEHM \& W.J.T. MITCHELL, «Pictorial versus Iconic Turn: Two Letters », Culture, Theory and Critique 50/2-3, 2009, pp. 103-121.

Pierre BOURDIEU (dir.), Un art moyen. Essai sur les usages sociaux de la photographie, Paris, Editions de Minuit, 1965.

Ralf BOHNSACK, Qualitative Bild- und Videointerpretation. Die dokumentarische Methode. Opladen/Farmington Hills, Barbara Budrich, 2009.

Roswitha BRECKNER, "Bildwahrnehmung - Bildinterpretation», Österreichische Zeitschrift für Soziologie, 37/2, 2012, pp. 143-164.

Horst BREDEKAMP, Stratégies visuelles de Thomas Hobbes. Le Léviathan, archétype de l'État moderne. Illustrations des cuvres et portraits, Paris, Editions de la Maison des sciences de l'homme, 2003.

Hanna BRINKMANN, Laura COMMARE, Helmut LEDER, Raphael ROSENBERG, «Abstract Art as a Universal Language? », Leonardo, 47 (3), 2014, pp. 256-257.

Stéphane DEBENEDETTI, «Visite occasionnelle du musée d'art et confort de visite : le rôle des compagnons ", in Olivier DONNAT \& Paul TOLILA (dir.), Le(s) Public(s) de la culture, vol. 2, Paris, Presses de Sciences Po, 2003, pp. 273-279.

Barbara B. DIEFENDORF, From penitence to charity: pious women and the Catholic Reformation in Paris, Oxford, Oxford University Press, 2004.

Jacqueline EIDELMAN \& Anne JONCHERY, «Sociologie de la démocratisation des musées ", Hermès, La Revue 2011/3 (n 61), pp. 52-60.

Jacqueline Eidelman, Anne Jonchery \& Lucile Zizi, Musées et publics: bilan d'une décennie (2002-2011), Paris, Direction générale des patrimoines, Département de la politique des publics, juin 2012.

Jacqueline EIDELMAN \& Nathalie RAGUET-CANDITO, «L'exposition La Différence et sa réception en Suisse, en France et au Québec: Le visiteur comme expert, médiateur et ethnologue », Ethnologie française, xxxii, 2, 2002, pp. 357-366.

Jacqueline EIDELMAN \& Mélanie ROUSTAN, «Les études de publics : Recherche fondamentale, choix de politiques et enjeux opérationnels", in Jacqueline EIDELMAN, Mélanie ROUSTAN \& Bernadette GOLDSTEIN (dir.), La Place des publics: De l'usage des études et recherches par les musées, Paris, La Documentation française (Musées-Mondes), 2008, pp. 15-37. 
Jessica EVANS et Stuart HALL, "What is Visual Culture ? », in Jessica EVANS, Stuart HALL (dir.), Visual Culture : the Reader, London, Sage, 1999, pp. 1-7.

Hana GOTTESDIENER, Le Public du musée national d'Art moderne en 1990 (étude sur la réception des collections permanentes), Paris, Centre Pompidou/Expo Média, 1990.

Hana GOTTESDIENER \& Jean DAVALLON (dir.), «La muséologie : 20 ans de recherches ", Cultures et Musées, Hors-série, 2013.

André GUNTHERT, L'image partagée, la photographie numérique, Paris, Éditions Textuel, 2015.

Max IMDAHL, Gesammelte Schriften, 3 vol, Gottfried Boehm et al. (dir.), Frankfurt am Main, Suhrkamp, 1996.

Solène KALENINE, " Mouvements oculaires et représentations sémantiques d'objets ", in Daniel DUBUISSON \& Sophie RAUX (dir.), A perte de vue. Les nouveaux paradigmes du Visuel, Dijon, Les Presses du réel, 2015, pp. 269-277.

Zoï KAPOULA, Quing YANG, Marine VERNET, Maria-Pia BUCCI, Louis-José LESTOCART, «Mouvements des yeux et perception de l'espace pictural : études des peintures de Francis Bacon et Pierro della Francesca », in Daniel DUBUISSON \& Sophie RAUX (dir.), A perte de vue. Les nouveaux paradigmes du Visuel, Dijon, Les Presses du réel, 2015, pp.293-303.

Atsuko KAWASHIMA \& Hana GOTTESDIENER, "Accrochage et perception des œuvres », Publics E musées, 13, 1998, pp. 149-173.

Hubert KNOBLAUCH, Jo REICHERTZ, Reiner KELLER (dir.), Kommunikativer Konstruktivismus (Wissen, Kommunikation und Gesellschaft), Wiesbaden, VS Verlag, 2012.

Christian METZ, Essais sur la signification au cinéma (I et II), Paris, Klincksieck, 19681972.

William John Thomas MITCHELL, Picture Theory, Chicago, University of Chicago Press, 1994.

Nicolas MILOVANOVIC \& Luc PIRALLA-HENG VONG (dir.), Le mystère Le Nain, Lens, Musée du Louvre-Lens, Paris, Editions Lienart, 2017.

Nicholas MIRZOEFF, An Introduction to Visual Culture, London, Routledge, 1999.

Lorenza MONDADA, “Understanding as an embodied, situated and sequential achievement in interaction", Journal of Pragmatics, 43, 2011, pp. 542-552.

Jan PACKER \& Roy BALLANTYNE, "Solitary vs. Shared Learning: Exploring the Social Dimension of Museum Learning », The Museum Journal, 48, 2, 2005, pp. 177-192.

Jean-Claude PASSERON, Le Raisonnement sociologique: un espace non poppérien de l'argumentation, Paris, Albin Michel, 2006.

Raphael ROSENBERG, «Blicke Messen. Vorschläge für eine empirische Bildwissenschaft », Jahrbuch der Bayerischen Akademie der Schönen Künste, 27, 2013, pp. 71-86.

Boris TRAUE, " Kommunikationsregime ", in Reiner KELLER, Hubert KNOBLAUCH, Jo REICHERTZ (dir.), Kommunikativer Konstruktivismus, Wiesbaden, SV, 2012, pp. 257-274.

Laurent SPARROW, "Les modèles théoriques de la perception visuelle à l'épreuve des images d'art : une étude oculométrique», in Nathalie DELBARD, Dork 
ZABUNYAN (dir.), L'œil mouvementé. Esthétique des images et oculométrie, Dijon, Les Presses du Réel, à paraître 2020.

Dirk VOM LEHN, Christian HEATH C. \& John HINDMARSH, «Exhibiting Interaction: Conduct and collaboration in museums and galleries ", Symbolic Interaction, 24(2), 2001, pp. 189-219.

RÉSUMÉ : Cet article présente une approche méthodologique de réception des peintures tenant compte des dimensions sociales et culturelles du regard. La méthode du tracé d'annotation se déploie à l'aide d'un outil numérique, Ikonikat (Ikonik Analysis Toolkit). Elle commence par une phase participative de récolte de données (des tracés d'annotation) et se continue par une combinaison d'analyses qualitatives et quantitatives de celles-ci. Les résultats d'une étude sur le terrain au musée du Louvre-Lens mettent en lumière différentes modalités de perception des peintures liées à des facteurs socioculturels et à l'influence de la muséographie. Ils font également état d'interprétations des publics qui interrogent la présentation des œuvres et suscitent un dialogue avec le savoir expert. Pour finir, ces résultats appellent à une prise en considération systématique de l'accompagnement de visite dans les études de réception des œuvres.

MOTS-CLÉS : Sociologie de la réception, Visual Studies, Museum Studies, Méthodes des sciences sociales 